be a consequence of the cluster structure $\mathrm{e}^{12}$ of the projectile.

In summary, we have measured the summed mass, $M_{s}$, of projectile fragments for 2.1-GeV/ nucleon ${ }^{20} \mathrm{Ne}+\mathrm{C}$ and ${ }^{20} \mathrm{Ne}+\mathrm{Mo}$. In the framework of the abrasion-ablation model the summed mass $M_{s}$ is the mass of the projectile residue before ablation takes place. The measured distributions of summed mass $M_{s}$ provide clear evidence of the validity of the abrasion process. The data are also consistent with an abrasion model modified to include a normal amount of nuclear trans parency. The data are inconsistent with the hypothesis of an abnormally large degree of nuclear transparency. In addition, the data provide evidence for several dominant fragmentation modes. One of the fragmentation modes consists of several roughly identical light fragments. This fragmentation mode may reflect the cluster structure of the projectile.

\footnotetext{
(a)Also at Lawrence Berkeley Laboratory and Space Sciences Laboratory, University of California, Berkeley, Cal. 94720.

${ }^{1}$ P. J. Lindstrom, D. E. Greiner, H. H. Heckman, B. Cork, and F. S. Bieser, Lawrence Berkeley Laboratory Report No. 3650, 1975 (unpublished).
}

${ }^{2}$ D. E. Greiner, P. J. Lindstrom, H. H. Heckman, B. Cork, and F. S. Bieser, Phys. Rev. Lett. $\underline{35}, 152$ (1975).

${ }^{3}$ J. B. Cumming, P. E. Haustein, R. W. Stoenner, L. Mausner, and R. A. Naumann, Phys. Rev. C 10, 739 (1974).

${ }^{4}$ D. J. Morrissey, W. R. Marsh, R. J. Otto, W. Loveland, and G. T. Seaborg, Phys. Rev. C 18, 1267 (1978).

${ }^{5}$ Y. P. Viyogi, T. J. M. Symons, P. Doll, D. E. Greiner, H. H. Heckman, D. L. Hendrie, P. J. Lindstrom, J. Mahoney, D. K. Scott, K. Van Bibber, G. D. Westfall, H. Weiman, H. J. Crawford, C. McParland, and C. K. Gelbke, Phys. Rev. Lett. 42 , 33 (1979).

${ }^{6}$ J. D. Bowman, W. J. Swiatecki, and C. F. Tsang, Lawrence Berkeley Laboratory Report No. 2908, 1973 (unpublished).

${ }^{7}$ D. J. Morrissey, L. F. Oliveira, J. O. Rasmussen, G. T. Seaborg, Y. Yariv, and Z. Fraenkel, Phys. Rev. Lett. 43, 1139 (1979).

${ }^{8}$ J. D. Stevenson, J. Martinis, and P. B. Price, to be published.

${ }^{9}$ The calculations in Fig. 1 are based on a Monte Carlo version of the abrasion model developed by the authors. A simple analytic version of the abrasion model is given in Ref. 6.

${ }^{10}$ A. Nadasen, P. Schwandt, P. P. Singh, W. W. Jacobs, A. D. Bacher, P. T. Debevec, M. D. Kaitchuck, and J. T. Meek, Phys. Rev. C 23, 1023 (1981).

${ }^{11} \mathrm{~J}$. W. Negele and K. Yazaki, Phys. Rev. Lett. 47, 71 (1981).

${ }^{12}$ D. Brink and J. J. Castro, Nucl. Phys. A216, 109 (1973).

\title{
Excited Multiplet Lines in Resonant Photoemission Spectra of Gd
}

\author{
F. Gerken, J. Barth, and C. Kunz \\ II. Institut für Experimentalphysik, Universität Hamburg, D-2000 Hamburg 50, Federal Republic of Germany \\ and Hamburger Synchrotronstrahlungslabor (HASYLAB) am Deutsches Elektronen Synchrotron, D-2000 \\ Hamburg 52, Federal Republic of Germany \\ (Received 20 July 1981)
}

\begin{abstract}
Resonant photoemission spectra of Gd metal in the photon energy range of the $4 d \rightarrow 4 f$ excitation show previously unobserved $4 f$ multiplet structures. These structures are identified as excited quintet lines by a comparison of a calculation in the fractional parentage scheme with the experimental curve, yielding excellent agreement. Our result provides new insight into the resonance interaction; it also implies an explanation of an unidentified resonance structure in SmS.
\end{abstract}

PACS numbers: $33.60 . \mathrm{Cv}, 32.80 . \mathrm{Hd}, 79.60 . \mathrm{Cn}$

The understanding of correlation effects in rareearth metals has been increased considerably by resonant photoemission in recent years. The decay processes of the excited states in the region of the $4 d \rightarrow 4 f$ "giant absorption resonance" influence the photoionization cross sections of the $4 f$ multiplets in the rare-earth metals (see, e.g., Lenth et al., ${ }^{1}$ Johansson et al., ${ }^{2}$ Allen etal., ${ }^{3}$ and Gerken etal. ${ }^{4}$ ). In this paper, we report the first observation of additional $4 f$ multiplet structure in the electron energy distribution curves (EDC) of $\mathrm{Gd}$ in the region of the $4 d \rightarrow 4 f$ excitation. It is 
well established that ordinary $4 f$ photoemission spectra are well described by the fractional parentage scheme ${ }^{5}$ which for Gd only allows the septet configuration. We prove that the additional structure originates from excited quintet configurations of the $4 f^{6}$ state reached by the Auger decay of the resonantly excited $4 d^{9} 4 f^{8}$ configurations. Our finding sheds new light on the mechanis $m$ of the resonance interaction.

Our measurements were performed in the HASYLAB with the FLIPPER monochromator using the synchrotron radiation of the storage ring DORIS in Hamburg. The photoelectrons were collected with a commercial double-pass cylindrical mirror analyzer. The geometry of the experiment is described by Kammerer et al. ${ }^{6}$ The Gd samples were evaporated in situ on stainlesssteel substrates under UHV conditions.

Three EDC's of Gd, measured in the region of the giant resonance, are shown in Fig. 1. Since the ground state of $\mathrm{Gd}$ is $\left(4 f^{7}\right)^{8} S_{7 / 2}$, for photon energies below the resonance the only final state for photoemission from the $f$ shell is $\left(4 f^{6}\right)^{7} F_{J}$ which leads to the dominant structure at $8.3-\mathrm{eV}$

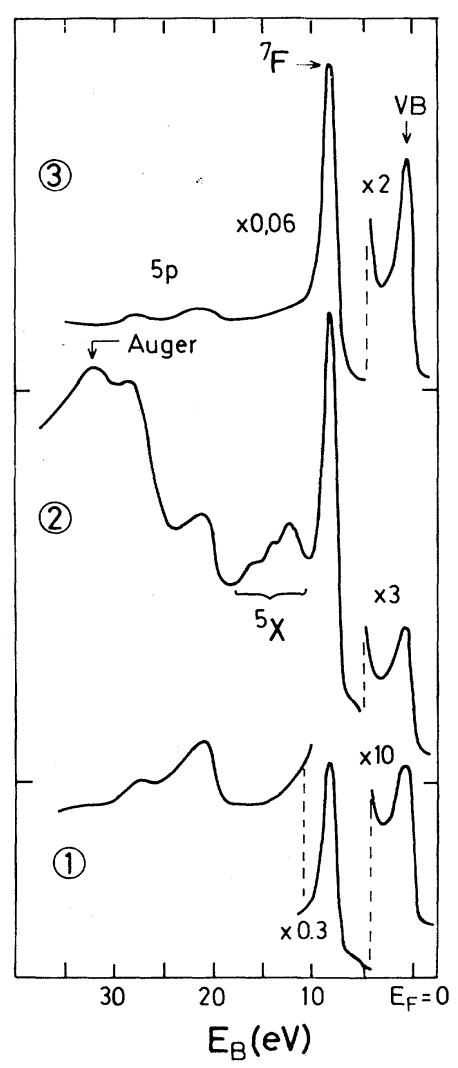

FIG. 1. EDC's of Gd; photon energies as indicated in Fig. 2. binding energy. The different $J$ components cannot be resolved. The intensity of this peak is correlated with the absorption structure and can be well fitted with a single Fano profile. ${ }^{4}$ In addition, curve 2 in Fig. 1 shows new structure in the binding-energy region of 10 to $20 \mathrm{eV}$. In Fig. 2 the intensity of this structure versus photon energy is given by the dashed curve. For photon energies in the maximum of the absorption the structures are dominated by the giant resonance of the ${ }^{7} F$ line, and the intensity could not be determined. For Eu metal, which has the same $4 f$ configuration as $\mathrm{Gd}$, we find equivalent results. This suggests that the details of the effect are dependent on the occupation of the $4 f$ shell.

We assign this structure to excited quintet configurations of the $4 f^{6}$ state which are accessible by the Auger decay of the resonantly excited $4 f^{8}$ configuration, as illustrated in Fig. 3. In the ground state Gd has seven $4 f$ electrons with parallel spins which form the configuration ${ }^{8} S_{7 / 2}$. In a "normal photoemission process" just one electron is kicked out and we get the final state $\left(4 f^{6}\right)^{7} F$. For photon energies in the giant resonance a $4 d$ $\rightarrow 4 f$ excitation leads to the configuration $4 d^{9} 4 f^{8}$. Following the Pauli principle the eighth electron must have opposite spin. The excited configuration can decay by direct recombination in two different channels:

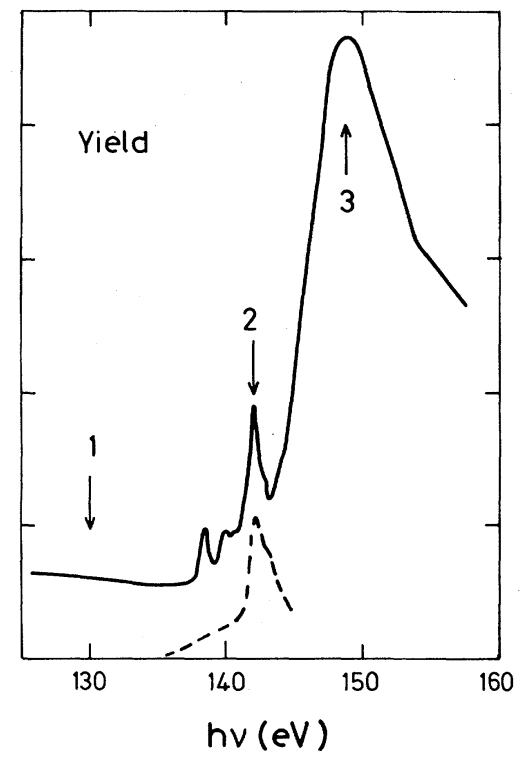

FIG. 2. Yield spectrum of Gd in the region of the $4 d \rightarrow 4 f$ excitation. The numbers refer to the EDC's of Fig. 1. The dashed curve gives the intensity of the quintet lines. 


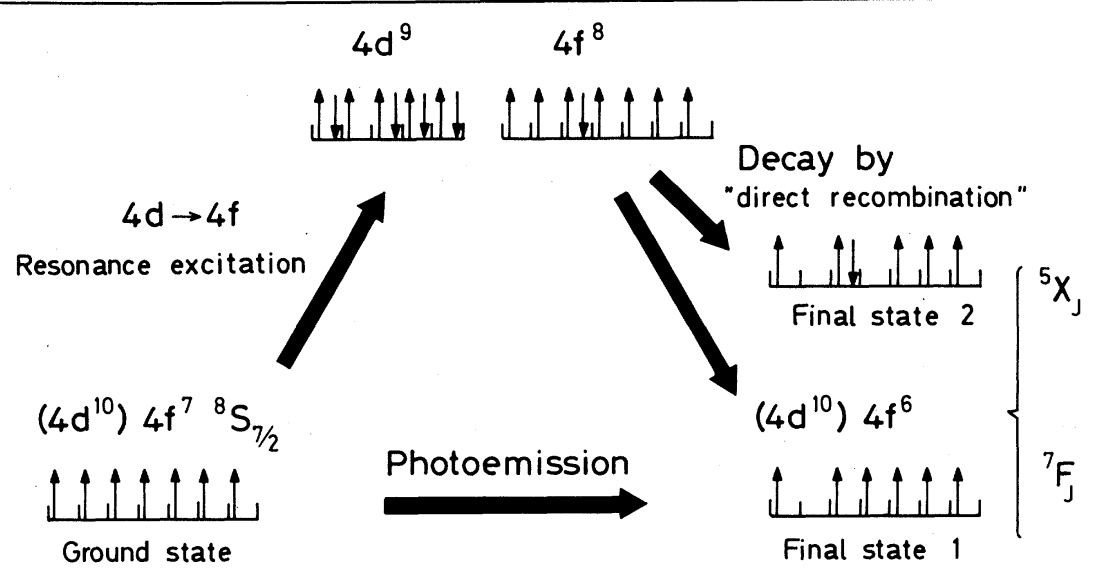

FIG. 3. Illustration of the $4 f$ photoemission process and the decay channels of the resonantly excited $4 d^{9} 4 f^{8}$ configuration in $\mathrm{Gd}$.

(1) The excited electron fills the $4 d$ hole and the energy is transferred to another $4 f$ electron or vice versa. This leads to the same final state as in normal photoemission. This process is responsible for the intensity variation of the ${ }^{7} F$ peak in the giant resonance.

(2) The electron with spin down stays in the $4 f$ shell and two other $4 f$ electrons take part in the Auger process. This leads to the final state ${ }^{5} X$, where $X$ stands for all possible angular momentum quantum rumbers. To reach these final states a spin flip of one electron is required either in the excitation of a $4 d$ electron to the $4 f$ shell or in the decay of the $4 d^{9} 4 f^{8}$ configuration. It is obvious from Fig. 2 that the intensity variation of the quintet lines does not follow the shape of the absorption curve but is mainly correlated with the fine structure indicated by number 2 in Fig. 2. Following Sugar's ${ }^{7}$ calculation for Gd a strong absorption line involving a spin flip appears at the onset of the giant resonance. Since the absorption fine structure is not too well reproduced, a refined calculation would be worthwhile.

In order to verify our assignment for the additional $4 f$ multiplet structure we compare the spectrum with a calculation in the fractional parentage scheme $e^{8}$ for the relative intensities of the quintet lines. Since the $4 d \rightarrow 4 f$ absorption even at a fixed photon energy may contain different excitations which do not all lead to quintet states we cannot expect to reproduce the correct septet to quintet intensity ratio with our calculation. We calculate the following processes: (1) excitation from $\left(f^{7}\right)^{8} S_{7 / 2}$ to $\left(f^{8}\right)^{7} F_{J} ;(2)$ decay from $\left(f^{8}\right)^{7} F_{J}$ to $\left(f^{7}\right)^{8} S_{7 / 2}$ and ${ }^{6} Y_{J}$, and decay from $\left(f^{7}\right)^{8} S_{7 / 2}$ (or $\left.{ }^{6} Y_{J}\right)$ to $\left(f^{6}\right){ }^{7} F_{J}\left(\right.$ or $\left.{ }^{5} X_{J}\right)$. $X$ and $Y$ again stand for all possible angular momentum quantum numbers. This calculation was done for every possible $J$ value of the different configurations with use of a formula given by Cox. ${ }^{9}$ In this approximation the influence of the $4 d$ hole is neglected.

The energy positions of the different levels of the $f^{6}$ configurations up to $5 \mathrm{eV}$ above the ground state ${ }^{7} F_{0}$ were taken from optical data of $\mathrm{Eu}^{3+}$ and a calculation in inter mediate coupling. ${ }^{10} \mathrm{We}$ calculated the higher-lying levels in LS-coupling approximation. A scaling factor is applied in order to account for the different nuclear charge of $\mathrm{Eu}^{3+}$ and $\mathrm{Gd}$ and for the screening of the valence electrons in the metal (Fig. 4). We then fold the calculated lines with asymmetric Doniach-Sunjic curves $^{11}$ to account for the fact that the atomic

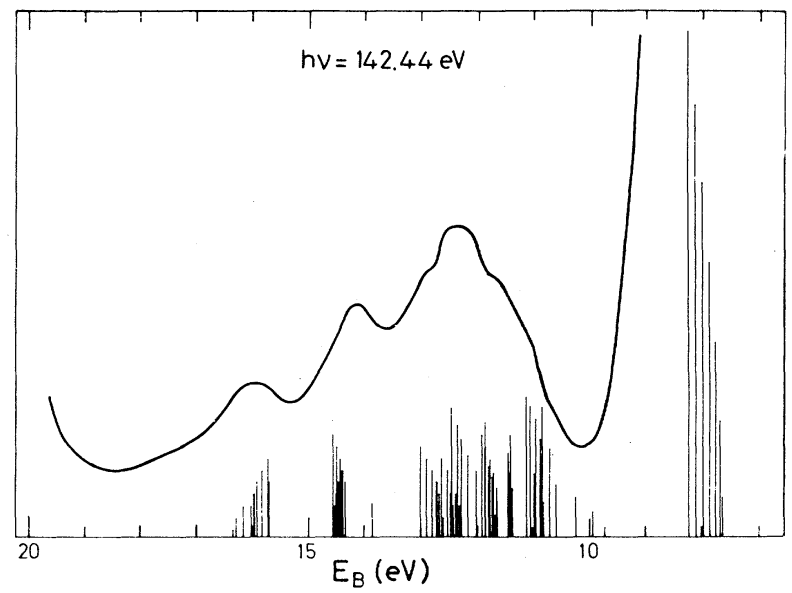

FIG. 4. Section of curve 2 in Fig. 1 showing the quintet states of $4 f^{6}$ on an enlarged scale (zero suppressed). The lines give the result of our calculation. 


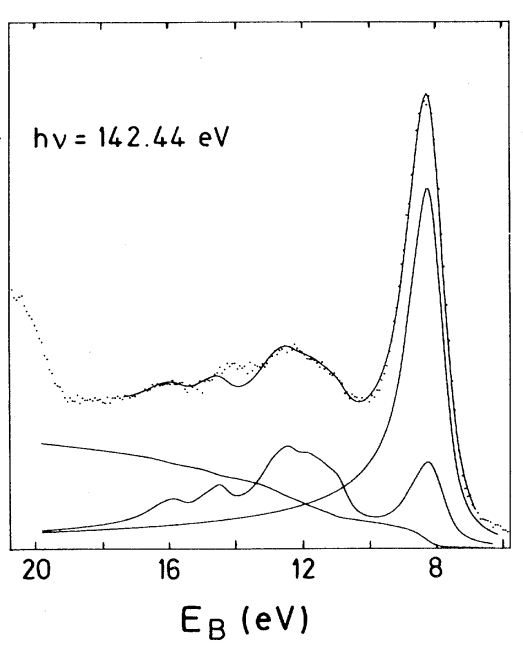

FIG. 5. Theoretical lines from Fig. 4 folded with asymmetric Doniach-Sunjic line shapes (solid curves) and compared with the measured EDC (points) as described in the text. The fit parameters are given in Ref. 6.

lines occur in a metallic sample. The experimental resolution was considered by a convolution with a Gaussian curve of $0.35 \mathrm{eV}$ full width at half maximum (FWHM) (lower curve in Fig. 5). Since we have strong evidence that the binding energy of the $4 f$ levels in $\mathrm{Gd}$ is lower in the bulk than at the surface ${ }^{6}$ this curve consists of two sets of lines with an energy difference of $0.48 \mathrm{eV}$ and a bulk to surface peak-height ratio of $1 / 0.4$.

Because the calculation does not give the correct ratio between the ${ }^{7} F$ and the ${ }^{5} X$ contribution we add a ${ }^{7} F$ part to the curve with an amplitude 4.25 times higher. Finally, we generate a background for the bulk contribution of the theoretical curve with a computer program which simulates the travel of the photoelectrons through the sample. The upper curve in Fig. 5 shows the sum of all discussed contributions in comparison with the measured curve.

As a result we see an excellent agreement between theory and experiment, particularly in the first part of the spectrum where the energy positions of these lines are available from intermediate-coupling calculations. For the scaling factor we determined the value of 0.97 . The equivalent results from Eu metal were analyzed in the same way as described for Gd. We determined a scaling factor of 0.93 . These values are much lower than those published by Lang, Baer, and $\operatorname{Cox}^{5}$ ( 1 for $\mathrm{Eu}$ and 1.26 for $\mathrm{Gd}$ ). While Lang, Baer, and Cox could only use the ${ }^{7} F$ peak from $\mathrm{x}$-ray photoelectron spectroscopy measure- ments with a FWHM of about $1.1 \mathrm{eV}$, we can use the large ${ }^{7} F$ and ${ }^{5} X$ multiplet splitting of about 10 $\mathrm{eV}$ to fit the scaling factor. Our scaling factor for Eu metal shows that the influence of the $6 \mathrm{~s}$ valence electrons in the screening of the nuclear charge is not negligible. In Gd the valence band contains a localized $5 d$ electron which obviously leads to a very effective screening of the additional nuclear charge compared with $\mathrm{Eu}$.

The excitation of new multiplet structure via resonant photoemission possibly provides also the explanation for a resonance structure reported for $\mathrm{SmS},{ }^{12}$ for which a satisfactory understanding is still lacking. The photoemission spectra of divalent $\mathrm{Sm}$ in $\mathrm{SmS}$ contain three maxima which are assigned to ${ }^{6} H,{ }^{6} F$, and ${ }^{6} P$. For a photon energy in the region of the fine structure preceding the giant resonance, Gudat, Alvarado, and Campagna $^{12}$ reported a very sharp resonance enhancement of the photoemission intensity at the ${ }^{6} P$ line only. This was interpreted as a Fano resonance of the ${ }^{6} P$ line. At the same time, the FWHM of the EDC structure at the ${ }^{6} P$ line goes through a maximum which was argued to reflect a minimum lifetime of the excited state.

This explanation must be strongly questioned since the FWHM of an EDC structure taking part in a resonance remains essentially unchanged because it reflects the lifetime of the final hole state, not that of the intermediately excited configuration. On the contrary, these observations quite naturally fit into the framework of new multiplet structure with lower multiplicity created by the Auger decay of an intermediately excited configuration as we outlined for Gd. Indeed, the energy positions of all quartet lines of $\mathrm{Sm}^{3+}$ are reported to coincide with the ${ }^{6} P$ state covering a range of $2.5 \mathrm{eV}^{13}$ The intensity profile obtained in Ref. 12 is similar to our finding for Gd (see Fig. 2, dashed curve).

We have shown that in the region of the giant resonances all details in the photoemission spectra are generated by the Auger decay of $4 d^{9} 4 f^{N+1}$ configurations. Our calculations within the fractional parentage scheme are a first step towards a quantitative theoretical description of the photoemission resonances. The inclusion of Auger matrix elements and the other parameters entering the Fano formalism now appears to be feasible also in the rare-earth metals, quite along the lines of the successful theory of the Mn $3 p \mathrm{ab}-$ sorption resonance by Davis and Feldkamp. ${ }^{14}$

${ }^{1}$ W. Lenth, F. Lutz, J. Barth, G. Kalkoffen, and 
C. Kunz, Phys. Rev. Lett. 41, 1185 (1978).

${ }^{2}$ L. I. Johansson, J. W. Allen, T. Gustafsson, I. Lindau, and S. B. M. Hagström, Solid State Commun. 28, 53 (1978).

${ }^{3} \mathrm{~J}$. W. Allen, L. I. Johansson, I. Lindau, and S. B. Hagström, Phys. Rev。B 21, 1335 (1980)。

${ }^{4}$ F. Gerken, J. Barth, K. L. I. Kobayashi, and C. Kunz, Solid State Commun. 35, 179 (1980).

${ }^{5}$ J. K. Lang, Y. Baer, and P. A. Cox, J. Phys. F 11, 121 (1981).

${ }^{6}$ R. Kammerer, J. Barth, F. Gerken, A. Flodström, and L. I. Johansson, to be published.
${ }^{7} J$. Sugar, Phys.Rev. B $\underline{5}, 1785$ (1972).

${ }^{8}$ G. Racah, Phys. Rev. 76, 1352 (1949).

${ }^{9}$ P. A. Cox, Struct. Bonding (Berlin) 24, 59 (1975).

${ }^{10}$ N. T. Carnall, P. R. Fields, and K. Rajnak, J. Chem. Phys. 49, 4450 (1968).

${ }^{11}$ S. Doniach and M. Sunjic, J. Phys. C 3, 285 (1970).

${ }^{12}$ W. Gudat, S。F. Alvarado, and M. Campagna, Solid State Commun. 28, 943 (1978).

${ }^{13}$ W. T. Carnall, P. R. Fields, and K. Rajnak, J。 Chem. Phys. 49, 4424 (1968).

${ }^{14}$ L. C. Davis and L. A. Feldkamp, Phys. Rev. A $\underline{17}$, 2012 (1978).

\title{
Resonance Features and Fine-Structure Effect in the Asymmetry of Polarized Electrons Scattered Inelastically from Mercury Atoms
}

\author{
K. Bartschat, G. F. Hanne, A. Wolcke, and J. Kessler \\ Physikalisches Institut, Universität Münster, D-4400 Münster, West Germany \\ (Received 6 May 1981)
}

\begin{abstract}
The energy dependence of the asymmetry in inelastic scattering of polarized electrons by $\mathrm{Hg}$ atoms (excitation of the $6^{3} P_{1}, 6^{3} P_{2}$, and $6^{1} P_{1}$ states) has been studied for a scattering angle of $90^{\circ}$ and collision energies between the threshold energies and $22 \mathrm{eV}$. Structures in the measured asymmetry have been found which are due to formation of negativeion states. Evidence has been found that interference between exchange and fine-structure interaction also causes a scattering asymmetry.
\end{abstract}

PACS numbers: $34.80 . D p$

Spin-polarization effects in elastic scattering of low-energy electrons from atoms and surfaces have been studied with increasing effort in the past fifteen years. The work on electron polarization in inelastic scattering is, however, very limited; indeed, ${ }^{1-3}$ one of the reasons being the greater difficulties of such experiments. This situation has recently been alleviated by the advent of techniques which yield rather high currents of polarized electrons with a small energy spread. ${ }^{4}$

Such sources of polarized electrons allow the spin-dependent interaction to be studied in an alternative way. Instead of using an unpolarized primary beam and measuring the spin polarization of the scattered electrons one can use a polarized primary beam with polarization $P$ and observe the left-right asymmetry $A$ of the scattered intensity. ${ }^{5}$

Measurements of the polarization of inelastically scattered electrons have been made earlier for the two channels of the $\mathrm{Hg}$ atom with energy losses 6.7 and $11.0 \mathrm{eV}$ at primary energies between 30 and $180 \mathrm{eV}^{1,2}$ The results may give the impression that there is a tendency of the polariza- tion effects to decrease as the primary energy is diminished. On the other hand it has been pointed out that in the energy range where exchange effects play a role, polarization effects caused by fine-structure interaction within the target may occur $^{6}$ which have not been observed so far. Contrary to "pure" exchange scattering which will not give rise to spin-polarization effects unless electrons or atoms are initially polarized,,$^{5}$ the combined effect of fine-structure interaction and exchange gives rise to spin polarization of the continuum electron. This is because atomic finestructure levels can be excited coherently via ("direct") collision without spin transfer and via exchange collision with spin transfer ("spin flip"). ${ }^{6}$ This "coherence" will vanish if the fine-structure states are not resolved, which is the description used most. ${ }^{\mathrm{b}}$ In addition, strong polarization effects in inelastic scattering may be expected at collision energies where negative-ion states $\mathrm{Hg}^{-}$ are temporarily formed. This can be concluded from earlier observations of resonances in $e-\mathrm{Hg}$ scattering ${ }^{7}$ and from studies of the polarization of elastically scattered electrons in the vicinity of such resonances. ${ }^{8}$ 